PROCEEDINGS OF THE

AMERICAN MATHEMATICAL SOCIETY

Volume 126, Number 3, March 1998, Pages 711-713

S 0002-9939(98)04604-8

\title{
ON A CLASSICAL THEOREM IN THE THEORY OF FOURIER INTEGRALS
}

\author{
ZOLTÁN SASVÁRI
}

(Communicated by J. Marshall Ash)

\begin{abstract}
In this note we give a short proof of a classical theorem in the theory of Fourier integrals.
\end{abstract}

We will say that an even, continuous function $f: \mathbb{R} \longrightarrow[0, \infty)$ with $f(0)=$ 1 is of Pólya-type if $f$ is convex on $(0, \infty)$ and $\lim _{t \rightarrow \infty} f(t)=0$. Titchmarsh ([6], Theorem 124) has proved (using a different terminology) that every Pólyatype function is the characteristic function of an absolutely continuous probability distribution. ${ }^{1}$ A proof of this result is essentially contained already in [4]. Esseen [1] and Pólya [5] were the first to point out the usefulness of Pólya-type functions in probability theory. Pólya also noted that the corresponding density is continuous except perhaps at zero. In their proofs, Titchmarsh and Pólya first show that

$$
p(x):=\frac{1}{2 \pi} \lim _{T \rightarrow \infty} \int_{-T}^{T} f(t) \cos x t \mathrm{~d} t \quad(x \neq 0)
$$

exists and is nonnegative. Then, applying Fourier's inversion theorem, they prove that $p$ is Lebesgue integrable and that

$$
f(t)=\int_{\mathbb{R}} p(x) \cos x t \mathrm{~d} x \quad(t \in \mathbb{R}) .
$$

The aim of the present note is to point out that the result above has a short proof that does not use inversion theorems. Moreover, $p$ can be given explicitly with a certain Lebesgue integral. Our proof is based on Fubini's theorem and the following simple fact (see e.g. Lukacs [3]):

A function $f$ is of Pólya-type if and only if it admits a representation

$$
f(t)=\int_{0}^{\infty} \psi(t / y) \mathrm{d} \nu(y)
$$

where $\nu$ is a probability distribution on $(0, \infty)$ and $\psi(t)=\max (0,1-|t|)$. The "if part" of this statement is trivial, while the "only if part" follows from the equation

$$
\int_{t}^{\infty}(1-t / y) \mathrm{d}\left[1-f(y)+y f^{\prime}(y)\right]=-t \int_{t}^{\infty} \frac{\mathrm{d}}{\mathrm{d} y}\left[\frac{f(y)}{y}\right] \mathrm{d} y=f(t), \quad t>0,
$$

Received by the editors March 4, 1996.

1991 Mathematics Subject Classification. Primary 42A38; Secondary 60E10.

${ }^{1}$ Since this theorem seems to have most of its applications in probability theory we use the terminology of that field. In textbooks on probability theory the result is usually referred to as Pólya's theorem. 
which can be proved by integrating by parts and using elementary properties of convex functions. Here $f^{\prime}$ and $\frac{\mathrm{d}}{\mathrm{d} y}$ denote right derivatives. From the equation above we also see that $\mathrm{d} \nu(y)=\mathrm{d}\left[1-f(y)+y f^{\prime}(y)\right]$.

For $y \in(0, \infty)$ and $x \in \mathbb{R}$ let

$$
K(x, y):=\frac{2}{\pi} \frac{\sin ^{2} y x / 2}{y x^{2}}=\frac{y}{2 \pi}\left(\frac{\sin y x / 2}{y x / 2}\right)^{2}
$$

if $x \neq 0$, and $K(0, y):=y / 2 \pi$. We will use the well-known fact (see e.g. [7], p. 251) that for any fixed $y$ the function $x \longrightarrow K(x, y)$ is a density and the corresponding characteristic function is $t \longrightarrow \psi(t / y)$. That is,

$$
\psi(t / y)=\int_{\mathbb{R}} K(x, y) \mathrm{e}^{\mathrm{i} t x} \mathrm{~d} x .
$$

By $\lambda$ we will denote the Lebesgue measure on $\mathbb{R}$.

Now we can prove the following theorem.

Theorem. Let $f$ be a Pólya-type function given by (1). Then:

(i) $f$ is the characteristic function of an absolutely continuous distribution with density

$$
p(x)=\frac{2}{\pi} \int_{0}^{\infty} \frac{\sin ^{2}(y x / 2)}{y x^{2}} \mathrm{~d} \nu(y)
$$

(ii) $p$ is finite and continuous on $\mathbb{R} \backslash\{0\}$;

(iii) $p$ is bounded if and only if

$$
\int_{0}^{\infty} y \mathrm{~d} \nu(y)<\infty
$$

Proof. (i) By Fubini's theorem (see e.g. [2])

$$
\begin{gathered}
1=\int_{0}^{\infty} 1 \mathrm{~d} \nu(y)=\int_{0}^{\infty} \int_{\mathbb{R}} K(x, y) \mathrm{d} x \mathrm{~d} \nu(y) \\
=\int_{\mathbb{R}} \int_{0}^{\infty} K(x, y) \mathrm{d} \nu(y) \mathrm{d} x=\int_{\mathbb{R} \times(0, \infty)} K(x, y) \mathrm{d} \lambda \times \nu(x, y),
\end{gathered}
$$

so that $K \in L^{1}(\lambda \times \nu)$. Moreover, the function

$$
p(x):=\int_{0}^{\infty} K(x, y) \mathrm{d} \nu(y) \quad(x \in \mathbb{R})
$$

is a probability density. Since $K \in L^{1}(\lambda \times \nu)$, we obtain from Fubini's theorem

$$
\begin{gathered}
\int_{\mathbb{R}} p(x) \mathrm{e}^{\mathrm{i} t x} \mathrm{~d} x=\int_{\mathbb{R}} \int_{0}^{\infty} K(x, y) \mathrm{d} \nu(y) \mathrm{e}^{\mathrm{i} t x} \mathrm{~d} x \\
=\int_{0}^{\infty} \int_{\mathbb{R}} K(x, y) \mathrm{e}^{\mathrm{i} t x} \mathrm{~d} x \mathrm{~d} \nu(y)=\int_{0}^{\infty} \psi(t / y) \mathrm{d} \nu(y)=f(t) .
\end{gathered}
$$

(ii) Using the inequalities $K(x, y) \leq 1 / 2 \pi(0<y \leq 1)$ and $K(x, y) \leq 2 / \pi x^{2}(y \geq$ $1)$, we see that $p(x)<\infty(x \neq 0)$. Moreover, in view of these inequalities, Lebesgue's dominated convergence theorem can be applied to show that $p$ is continuous on $\mathbb{R} \backslash\{0\}$. 
(iii) If $\int y \mathrm{~d} \nu(y)<\infty$ then, using the inequality $K(x, y) \leq y / 2 \pi=K(0, y)$, we see that $p(x) \leq p(0)<\infty$ holds for every $x$. Assume now that $p$ is bounded. Then, by Fatou's lemma,

$$
\begin{aligned}
& \infty>\liminf _{x \rightarrow 0} p(x)=\liminf _{x \rightarrow 0} \int_{0}^{\infty} K(x, y) \mathrm{d} \nu(y) \\
& \geq \int_{0}^{\infty} \liminf _{x \rightarrow 0} K(x, y) \mathrm{d} \nu(y)=\frac{1}{2 \pi} \int_{0}^{\infty} y \mathrm{~d} \nu(y)
\end{aligned}
$$

completing the proof.

\section{REFERENCES}

[1] Esseen, C.-G.: Fourier analysis of distribution functions. Acta Math. 77, 1-125(1944). MR $7: 312 \mathrm{a}$

[2] Hewitt, E., Ross, K. A.: Abstract Harmonic Analysis I. New York: Springer-Verlag 1963. MR 28:158

[3] Lukacs, E.: Characteristic Functions. London: Griffin 1960. MR 23:A1392

[4] Pólya, G.: Über die Nullstellen gewisser ganzer Funktionen. Math. Zeitschrift, 2, 352383(1918).

[5] Pólya, G.: Remarks on characteristic functions. Proc. First Berkeley Conf. on Math. Stat. and Prob., 115-123, Berkeley: Univ. of Calif. Press 1949. MR 10:463c

[6] Titchmarsh, E. C.: Introduction to the Theory of Fourier Integrals. Oxford: Clarendon Press 1937. MR 89c:42002 (3rd ed.)

[7] Zygmund, A.: Trigonometric Series, Vol. II., Cambridge: University Press 1959. MR 21:6498

Department of Mathematics, Technical University of Dresden, Mommsenstrasse 13, 01062 Dresden, Germany

E-mail address: sasvari@math.tu-dresden.de 